\title{
Dos nuevos microRNAs (miRNAs) identificados en ovario bovino
}

\author{
Two new miRNAs identified in bovine ovary
}

\author{
LA Muñoz-Bañales ${ }^{\mathrm{a}}$, B Sánchez-Ramírez ${ }^{\mathrm{a}}$, E González-Rodríguez ${ }^{\mathrm{b}}$, V Moreno-Brito , \\ C González-Horta ${ }^{\mathrm{a}}$, ME Burrola-Barraza ${ }^{\mathrm{b}}$
}

\author{
aPrograma de Maestría en Ciencias en Biotecnología, Facultad de Ciencias Químicas, \\ Universidad Autónoma de Chihuahua, Chihuahua, México. \\ ${ }^{\text {b}}$ Facultad de Zootecnia y Ecología, Universidad Autónoma de Chihuahua, Chihuahua, México. \\ 'Facultad de Medicina, Universidad Autónoma de Chihuahua, Chihuahua, México.
}

\begin{abstract}
SUMMARY
The microRNAs (miRNAs) that participate in regulating oocyte growth and oocyte-embryo transition must be identified and characterised to understand maturation, fertilization and embryo development mechanisms in bovine reproduction. With the purpose of identifying new miRNAs that may play a role in oocyte maturation and early embryogenesis, a miRNAs genome library from bovine ovarian was developed. After analysing concatamers from 40 clones, two new miRNAs were identified and labeled bta-mir-8548 and bta-mir-8549. These miRNAs showed a stable stem-loop structure in accordance with established miRBase parameters. bta-mir-8548 was found at chromosome 8 in an intronic region between exons 1 and 2 of the MRSA gene (methionine sulfoxide reductase) suggesting that it may play a role in regulating oxidative stress during oocyte maturation. In the same way, bta-mir-8549 was found at chromosome 7 in the intergenic region between genes tRNA-Cys and GBPg5. The mature form of bta-mir-8549 showed the conserved sequence of the btalet-7 family, which is associated with oocyte maturation. With the purpose of locate the correct place of bta-mir-8549 into the family of miRNA bta-let-7 a phylogenetic study was conducted. The cladogram generated from genetic distances between the various bta-let-7 miRNAs showed that bta-mir-8549 was located monophyletically with bta-lec-7c, while the rest of the bta-let-7 miRNAs were located in different clades. It was concluded that bta-mir-8548 and bta-mir-8549 are new bovine ovarian miRNAs that may act on the mechanisms regulating oocyte maturation and embryonic development.
\end{abstract}

Key words: microRNAs, oocyte, embryonic development.

\section{RESUMEN}

Para entender los mecanismos de maduración, fertilización y desarrollo embrionario en el ganado bovino, es necesario la identificación y caracterización de microRNAs (miRNAs) involucrados en la regulación del crecimiento del ovocito y la transición ovocito-embrión. Con el objetivo de identificar nuevos miRNAs, con una posible función dentro del proceso de maduración del ovocito y de embriogénesis temprana, se realizó una librería genómica con miRNAs provenientes de ovarios de vacas de rastro. Al analizar los concatámeros de 40 clonas se identificaron dos nuevos miRNAs, bta-mir-8548 y bta-mir-8549, cuyos pre-miRNA mostraron una estructura tallo-burbuja estable, según los parámetros establecidos por el miRBase. El bta-mir-8548 se ubicó en el cromosoma 8 en una región intrónica entre los exones 1 y 2 del gen MRSA (metionina sulfóxido reductasa), sugiriendo que puede tener una función en la regulación del estrés oxidativo durante la maduración del ovocito. El bta-mir-8549 se ubicó en el cromosoma 7 en la región intergénica entre los genes tRNA-Cys y GBPg5. La forma madura de este miRNA mostró la secuencia conservada propia de la familia de miRNAs bta-let-7, los cuales están relacionados con los procesos de maduración del ovocito. Para ubicar el lugar de bta-mir-8549 dentro de la familia de miRNAs bta-let-7 se realizó un estudio filogenético. El cladograma generado a partir de las distancias genéticas entre los miRNAs bta-let-7 indicó que bta-mir-8549 se situó de forma monofilética con bta-let-7c, mientras que el resto de los bta-let-7 quedaron ubicados en clados diferentes. En conclusión, bta-mir-8548 y bta-mir-8549 son nuevos miRNAs aislados de ovario bovino los cuales podrían actuar en los mecanismos de regulación de la maduración del ovocito y durante el desarrollo embrionario.

Palabras clave: mRNAs maternos, ovocito, desarrollo embrionario.

\section{INTRODUCCIÓN}

La activación del genoma embrinario ocurre dependiendo de la especie en diferentes etapas del desarrollo embrionario, en el ganado bovino esta activación se presenta en el

Aceptado: 17.10 .2013 .

* Perif. R. Almada km. 1, C.P. 31453 Chihuahua, México; mburrola1@uach.mx embrión de 8 a 16 células (Telford y col 1990), a diferencia del ratón donde ocurre en la etapa de 2 células (Schultz 1993) o del humano donde se presenta en la etapa embrionaria de 8 células (Telford y col 1990). Durante este proceso existe una degradación de RNAs mensajeros (mRNAs) maternos que fueron almacenados en el ovocito durante la ovogénesis (Bettegowda y col 2008). Esta degradación de transcritos maternos es un evento esencial para que la embriogénesis temprana se desarrolle de forma adecuada ( $\mathrm{Li}$ 
y col 2010). En especies como el ratón (Tang y col 2007), pez cebra (Giraldez y col 2006) y pez trucha arcoíris (Ramachandra y col 2008), se relaciona con la acción de los microRNAs (miRNAs) que al reconocer el 3'UTR de un mRNA materno promueven su degradación o bloquean su traducción. Los miRNAs son RNAs con una longitud de 22 a 24 nucleótidos de largo, están codificados dentro de regiones intergénicas y regiones intrónicas. Los primeros se expresan bajo la acción de la RNA polimerasa II y se denominan microRNA primario (pri-miRNA), son procesados por un complejo ribonucleico proteico compuesto por la enzima Drosha y la proteína DGCR8 que cortan al pri-miRNA y lo dejan en una estructura de tallo-burbuja que se denomina microRNA precursor (pre-miRNA). Los miRNAs que se encuentran codificados en regiones intrónicas se expresan de acuerdo al gen donde se encuentren, formando lo que se conoce como mirtron, y son liberados durante el empalme de exones en forma de tallo burbuja. Sea cual sea la vía de procesamiento del tallo-burbuja, el resultado es el mismo un pre-miRNA que es exportado al citoplasma a través de la proteína exportina 5 , donde es procesado por la enzima DICER, para obtener 2 microRNAs de $20 \mathrm{pb}$, a los cuales se les denomina miRNAs maduros. Una de las cadenas se selecciona para funcionar como un miRNA maduro, mientras que la otra cadena es degradada. En ocasiones, las dos hebras de la horquilla del pre-miRNA dan lugar a miRNAs maduros (Bushati y Cohen 2007). Posteriormente, los miRNAs son ensamblados en un complejo de ribonucleoproteínas llamado complejo de silenciamiento inducido por miRNA (miRISCs) conformado por proteínas Argonautas (AGO). Una vez que los miRNAs son ensamblados en el miRISCs, los nucleótidos de la posición 2 al 8 en el extremo $5^{\prime}$ del miRNA participan en el reconocimiento del extremo 3'UTR del mRNA blanco. Cuando se lleva a cabo la unión del miRNA con el 3'UTR del RNAm blanco, las proteínas AGO reprimen la expresión del MRNA blanco, de forma tal que si el acoplamiento es completo se induce una degradación endonucleótica, o bien, si el acoplamiento es desigual se inhibe la traducción y se promueve una degradación por exonucleasas (Filipowicz y col 2008).

En el modelo bovino se han aislado 662 miRNAs, reportados en el miRBase (Kozomara y Griffiths-Jones 2011). Sin embargo, es poca la información que se encuentra sobre la participación de los miRNAs en los proceso de ovogénesis y embriogénesis temprana. Con el propósito de identificar nuevos miRNAs relacionados, se generó una librería de miRNAs de ovario bovino, de la cual se reporta la identificación de dos nuevos miRNAs con una probable participación en estos procesos.

\section{MATERIAL Y MÉTODOS}

\section{GENERACIÓN DE LA LIBRERÍA DE miRNAS}

A partir de 10 ovarios de vacas del rastro municipal, se cortó de cada uno $150 \mathrm{mg}$ de tejido que incluyera un folículo antral de $8 \mathrm{~mm}$ de diámetro. De cada tejido se obtuvo RNA total mediante el reactivo TRIzol ${ }^{1}$. El total del RNA obtenido de cada muestra se colocó en un solo microtubo y se procesó de acuerdo a las instrucciones del fabricante del kit miRCat ${ }^{2}$. Brevemente, $18 \mu \mathrm{g}$ de RNA total fueron fraccionados en un gel de bis/acrilamida (19:1) al $15 \%$ con urea $7 \mathrm{M}$ a $90 \mathrm{~V}$ durante $3 \mathrm{~h}$. Al término el gel se tiñó con SYBRGreen durante $30 \mathrm{~min}$. Se llevó al fotodocumentador y se cortó la banda de 20 nt, la cual se homogenizó utilizando un pistilo y agua libre de RNAsas a $70^{\circ} \mathrm{C}$ durante $10 \mathrm{~min}$. El RNA de $20 \mathrm{nt}$ (miRNAs) se purificó en columnas de filtración en gel DTR $^{3}$. Los miRNAs aislados se ligaron, en ausencia de ATP, por acción de la enzima T4 RNA ligasa ( $1 \mathrm{U} / \mu \mathrm{L})$ por $2 \mathrm{~h}$ a $22^{\circ} \mathrm{C}$. Después de limpiar el miRNA-adaptador '3, se sometió de nuevo a electroforesis en un gel de bis/ acrilamida (19:1) al $15 \%$ y $7 \mathrm{M}$ de urea a $90 \mathrm{~V}$ durante 3 h. Posteriormente el gel se tiñó con SYBRGreen ${ }^{\circledR}$ durante $30 \mathrm{~min}$, se cortó la banda correspondiente a $40 \mathrm{nt}$ y se purificó en columnas DTR para ligar el adaptador 5 , en presencia de ATP, por medio de la enzima T4 RNA ligasa $(5 \mathrm{U} / \mu \mathrm{L})$ por $2 \mathrm{~h}$ a $22^{\circ} \mathrm{C}$. La mezcla, adaptador 5'-mirRNA-adaptador' 3 , fue purificada con glucógeno y acetato de sodio, para posteriormente someterse a transcripción reversa con la enzima SuperScript III RT ${ }^{1}$ (200 $\mathrm{U} / \mu \mathrm{L}$ ), utilizando un oligonucleótido complementario a la secuencia del adaptador ' 3 . El cDNA resultante se sometió a una PCR convencional con $5 \mathrm{U} / \mu \mathrm{L}$ de la enzima Taq Platinium ${ }^{1}$ y oligonucleótidos complementarios a las secuencias de ambos adaptadores. Se siguieron las siguientes condiciones de amplificación: temperatura de inicio de $95^{\circ} \mathrm{C}$ por $10 \mathrm{~min}$, seguido de 35 ciclos con una temperatura de desnaturalización de $95^{\circ} \mathrm{C}$ por $30 \mathrm{seg}$, temperatura de alineación de $52^{\circ} \mathrm{C}$ por $30 \mathrm{seg}$, elongación de $72^{\circ} \mathrm{C}$ por $30 \mathrm{seg}$ y un último ciclo de extensión final a $72^{\circ} \mathrm{C}$ por $5 \mathrm{~min}$. Los productos de PCR se limpiaron con fenol:cloroformo:alcohol isoamílico $(25: 24: 1)$ y acetato de sodio. Los amplicones se digirieron con la enzima Ban I y se ligaron con la enzima T4 DNA ligasa $(30 \mathrm{U} / \mu \mathrm{L})$ para obtener los concatámeros. Posteriormente, estos se adenilaron en sus extremos, se clonaron en el vector PCR 4-TOPO ${ }^{1}$ y se transformaron bacterias competentes One Shot TOP10 $10^{1}$, las que se sembraron en cajas con agar Louria Broth con Kanamicina $(50 \mu \mathrm{g} / \mathrm{mL}$ ) incubando toda la noche a $37^{\circ} \mathrm{C}$. Colonias individuales (clonas) se sembraron en $5 \mathrm{ml}$ de medio líquido Louria Broth con Kanamicina $(50 \mu \mathrm{g} / \mathrm{mL})$ durante $12 \mathrm{~h}$ a $37^{\circ} \mathrm{C}$. Posteriormente se extrajo el DNA plasmídico por lisis alcalina y se identificaron los insertos mediante PCR con los oligonucleótidos universales T3 y T7.

\footnotetext{
${ }^{1}$ Invitrogen

${ }^{2}$ IDT, DNA Caralville, IA

${ }^{3}$ Edge Bio
} 


\section{ANÁLISIS BIOINFORMÁTICO}

Para encontrar la región correspondiente a los concatámeros, la secuencia de cada clona se alineó con la secuencia completa del vector PCR4-TOPO, y se excluyó del análisis la secuencia correspondiente a dicho vector. Posteriormente, en la región del concatámero se buscó la secuencia correspondiente al conector, el cual es la secuencia resultante entre la unión del adaptador 5’ de un miRNA más el adaptador 3' de otro miRNA. Las secuencias nucleotídicas que se encontraron entre cada conector correspondieron a probables miRNAs maduros. Cada secuencia de probables miRNAs maduro fue analizada en el programa miRBase ${ }^{4}$ (Ambros y col 2003), para buscar alguna homología con miRNAs ya reportados en Bos taurus. Los probables miRNAs maduros que coincidieron en un $100 \%$ de identidad con un miRNA de Bos taurus en el miRBase, se consideraron ya reportados. Aquellos miRNAs maduros que no coincidieron con Bos taurus pero que fueron idénticos con miRNAs de otras especies, se consideraron nuevos. Los probables miRNAs maduros que no hibridaron con ningún miRNA del miRBase, fueron analizados en el Blast del $\mathrm{NCBI}^{5}$ para buscar identidad con RNAs de transferencia (tRNA), RNAs ribosomales (rRNA), RNAs nucleares pequeños (snoRNA) y secuencias mitocondriales, aquellos que no tuvieron relación con este tipo de RNAs fueron considerados nuevos. El pre-miRNA de cada miRNA maduro nuevo, fue localizado in silico usando la secuencia de miRNA en el Blast del $\mathrm{NCBI}^{5}$ sobre el genoma de Bos taurus (NW_003104074.1 Bos taurus_UMD_3.1), para localizar su posición cromosomal y encontrar las regiones flanqueantes del miRNA maduro. La predicción de la estructura secundaria (tallo-burbuja) de los pre-miRNAs se analizó por medio del algoritmo de Zuker en el programa mfold $^{6}$ (Zuker 2003) y se confirmó de acuerdo a los parámetros establecidos en el programa miRNAminer ${ }^{7}$ (Artzi y col 2008). Para identificar la región conservada del miRNA maduro aislado, se buscó homología en el miRBase para el pre-miRNA tanto en Bos taurus, como en humano y ratón. Posteriormente con las secuencias homólogas se realizó un alineamiento múltiple en el programa CLUSTAL W (Thompson y col 1994). A aquellos miRNAs maduros que presentaron una homología en Bos taurus con una familia en particular de miRNA, se les realizó un análisis filogenético con el paquete PHYLIP (Retief 2000). La distancia genética entre las secuencias se obtuvo con el modelo de 2 parámetros de Kimura (Kimura 1980) y el árbol filogenético se construyó por el método Neighbour-joining (Saitou y Nei 1987) con un remuestreo (bootstrap) de 1000 réplicas.

\footnotetext{
$4 \quad$ http://www.mirbase.org/

http://blast.ncbi.nlm.nih.gov/Blast.cgi

6 http://mfold.rna.albany.edu/?q=mfold

http:/groups.csail.mit.edu/pag/mirnaminer/
}

\section{RESULTADOS}

De la librería de miRNAs que se generó a partir de un enriquecido de RNAs de aproximadamente $20 \mathrm{nt}$ de longitud procedentes de ovarios bovinos, se obtuvieron 40 clonas las cuales se denominaron OV más el número de la clona; se les extrajo DNA plasmídico y se analizó por PCR para confirmar insertos. Para identificar las secuencias correspondientes al vector y a los conectores, los insertos se secuenciaron y analizaron con el programa "Basic Local Aligment Search Tool" (BLAST) del Centro Nacional para la Información Biotecnológica (NCBI, por sus siglas en inglés) de la Biblioteca Nacional de Medicina de Estados Unidos. En la figura 1 se muestra el análisis de la clona OV6, todas las clonas se analizaron de la misma forma. Del total de clonas analizadas, solo 12 mostraron secuencias propias de miRNAs (cuadro 1) y de estas se obtuvieron un total de 28 miRNAs maduros cuyos tamaños oscilaron entre 17 y 24 nucleótidos. La mayoría de los miRNAs maduros encontrados ya estaban reportados en la base de datos miRBase, solo los miRNAs OV1.4 y OV6.3 tuvieron una homología inferior al $100 \%$ con los miRNAs bta-miR-1185 y bta-let-7c, respectivamente (cuadro 1), lo que indicó que se trataba de nuevos miRNAs. De acuerdo a la nomenclatura establecida por el miRBase estos nuevos miRNAS fueron denominados como bta-mir-8548 y bta-mir-8549. Una condición indispensable para poder incluir un nuevo miRNA en la base de datos del miRBase, es contar con la secuencia completa del pre-mirRNA. Ya que los miRNA que se obtuvieron de la librería son miRNAs maduros, fue necesario identificar la secuencia del pre-miRNA in silico en el genoma de Bos taurus de la base de datos del NCBI. Para ubicar las regiones flanqueantes del miRNA maduro se analizaron, de acuerdo a lo establecido para la nomenclatura del miRBase, 5 bases hacia el extremo 5 y hacia el extremo 3' lo que faltara para completar una región de 70 a 85 bases. De esta forma se ubicó el btamir-8548 en el cromosoma 8 (figura 2A), mientras que el bta-mir-8549 se localizó en el cromosoma 7 (figura 2B). La secuencia de estos nuevos pre-miRNAs (cuadro 2) presentó, de acuerdo al programa mfold, una estructura tallo-burbuja estable (figura 3).

El bta-mir-8548 resultó ser un miRNA del tipo mirtrón, ya que se ubicó dentro de un intrón entre los exones 1 y 2 del gen MRSA (figura 2A). Esta condición de mirtrón, implica que este miRNA se expresa de acuerdo a la forma de expresión de MRSA, por lo cual su función debe estar muy relacionada con la de este gen. Por su parte, el bta-mir-8549 mostró un $90 \%$ de homología con el miRNA bta-let-7c y se localizó de forma intergénica entre los genes tRNA-Cys y GBPg5 (figura 2B). Dado que en el ganado bovino, la mayoría de los miRNAs pertenecientes a la familia Let-7 ya han sido reportados; el bta-mir-8549 se comparó con dichas secuencias mediante un alineamiento múltiple. Los resultados mostraron 


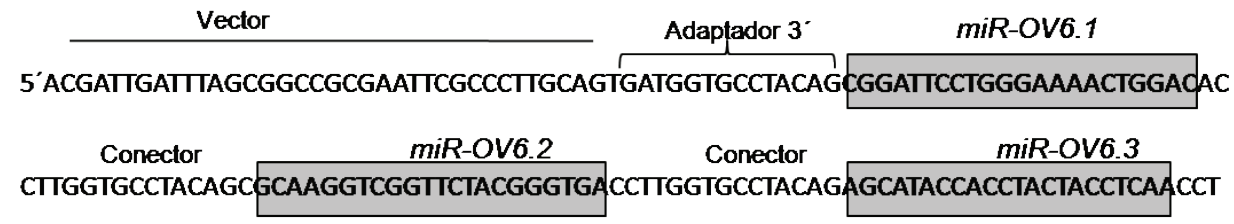

$\underbrace{\text { TGGTGCACCATCAATCAAGGGCGAATTCGTT'3 } 3}_{\text {Adaptador } 5^{\circ}}$

Figura 1. Análisis de la secuencia de la Clona OV6. Se muestran las secuencias que corresponden al vector, al adaptador y a los conectores. Los recuadros muestran las secuencias que corresponden a los miRNAs presentes en la clona OV6.

Sequence Analysis of Clone OV6, showing the sequences corresponding to the vector, the adapter and the connectors. The boxes show the sequences corresponding to the miRNAs present in the clone OV6.

Cuadro 1. Secuencias de miRNAs aislados y porcentaje de identidad encontrada con miRNAs homólogos reportados en la base de datos miRBase.

Sequences of miRNAs isolated and identity percent found with homologous miRNAs that have been reported in miRBase database.

\begin{tabular}{|c|c|c|c|c|c|c|}
\hline CLONA & miRNA & SECUENCIA $(5-3)$ & TAMAÑO & HOMOLOGIA (B. taurus) & $\%$ IDENTIDAD & NO. ACCESO MIRBASE \\
\hline \multirow{4}{*}{ OV1 } & OV1.1 & CAAAGAATTCTCCTIITGGGC & 21 & bta-miR-186 & 100 & MIMAT0003818 \\
\hline & OV1.2 & AACCCGTAGATCCGATCTTGT & 21 & bta-miR-99a & 100 & MIMAT0003537 \\
\hline & OV1.3 & GACCCGTAGATCCGATCTTGT & 21 & bta-miR-99a & 100 & MIMAT0003537 \\
\hline & OV1.4 & AAGGATTACCTTGGTGC & 17 & bta-miR-1185 & 43 & MIMAT0009973 \\
\hline \multirow{3}{*}{ OV2 } & OV2.1 & CAAAGAATTCTCCTITGGGC & 21 & bta-miR-186 & 100 & MIMAT0003818 \\
\hline & OV2.2 & AACCCGTAGATCCGATCTTGT & 21 & bta-mir-99a & 100 & MIMAT0003537 \\
\hline & OV2.3 & GACCCGTAGATCCGATCTTGT & 21 & bta-miR-99a & 100 & MIMAT0003537 \\
\hline OV5 & OV5.1 & TTCAAGTAATCCAGGATAGGCT & 22 & bta-miR-26a & 100 & MIMAT0003516 \\
\hline \multirow{3}{*}{ OV6 } & OV6.1 & GTCCAGTIITCCAGGAATCCG & 22 & bta-miR-145 & 100 & MIMAT0003542 \\
\hline & OV6.2 & CACCCGTAGAACCGACCTTGCG & 22 & bta-miR-99b & 100 & MIMAT0004345 \\
\hline & OV6.3 & TGAGGTAGTAGGTGGTATGCT & 21 & bta-let-7c & 90 & MIMAT0004332 \\
\hline OV8 & OV8.1 & AACCCGTAGATCCGATCTTGT & 21 & bta-miR-99a & 100 & MIMAT0003537 \\
\hline \multirow{5}{*}{ OV9 } & OV9.2 & TTGTACCGTGAGTAATAATGCG & 22 & bta-miR-126 & 100 & MIMAT0003540 \\
\hline & OV9.3 & TCCCTGAGACCCTAACTTGTGA & 22 & bta-miR-125b & 100 & MIMAT0003539 \\
\hline & OV9.4 & TTCAAGTAATCCAGGATAGGCT & 22 & bta-miR-26a & 100 & MIMAT0003516 \\
\hline & OV9.5 & TACCCTGTAGAACCGAATITGT & 22 & bta-miR-10b & 100 & MIMAT0003839 \\
\hline & OV9.7 & TCCCTGAGACCCTAACTTGTGA & 22 & bta-miR-125b & 100 & MIMAT0003539 \\
\hline \multirow{2}{*}{ OV13 } & OV13.1 & AACATTCAACGCTGTCGGTGAGT & 22 & bta-miR-181a & 100 & MIMAT0003543 \\
\hline & OV 13.2 & AACCCGTAATCCGATCTTGTGT & 22 & bta-miR-99a & 100 & MIMAT0003537 \\
\hline OV18 & OV18.1 & TGTAAACATCCCCGACTGGAAGCT & 24 & bta-miR-30d & 100 & MIMAT0003533 \\
\hline \multirow{2}{*}{ OV20 } & OV20.1 & AACATTCAACGCTGTCGGTGAGT & 23 & bta-miR-181a & 100 & MIMAT0003543 \\
\hline & OV20.3 & TCCCTGAGACCCTAACTTGTG & 21 & bta-miR-125b & 100 & MIMAT0003539 \\
\hline \multirow{2}{*}{ OV21 } & OV21.1 & CCTCAGTCAGCCTTGTGGATGT & 22 & bta-miR-3431 & 100 & MIMAT0017394 \\
\hline & OV21.2 & AACCCGTAGATCCGATCTTGTGA & 23 & bta-miR-99a & 100 & MIMAT0003537 \\
\hline \multirow{2}{*}{ OV22 } & OV22.1 & CCTCAGTCAGCCTTGTGGATGT & 22 & bta-miR-3431 & 100 & MIMAT0017394 \\
\hline & OV22.2 & AACCCGTAGATCCGATCTTGTGA & 23 & bta-miR-99a & 100 & MIMAT003537 \\
\hline \multirow{2}{*}{ OV31 } & OV31.1 & AACCCGTAGATCCGATCTTGT & 21 & bta-miR-99a & 100 & MIAMT003537 \\
\hline & OV 31.2 & AACCCGTAGATCCGATCTGTGT & 21 & bta-miR-99a & 100 & MIAMT003537 \\
\hline
\end{tabular}

que este nuevo miRNA presenta la secuencia consenso 5'GAGGUAG'3 en la misma posición que el resto de los bta-let-7 reportados (figura 4). Ya con la secuencia de toda la familia de miRNAs Let-7 de bovino alineada, se procedió a realizar un análisis filogenético para determinar la distancia genética existente entre estos miRNAs. El cladograma indicó que en el bovino la familia de miRNA Let-7 está conformada por tres grupos. bta-mir-8549 se situó de forma monofilética estrechamente relacionada con bta-let-7c, mientras que el resto de los bta-let-7 quedaron ubicados en clados diferentes, distantes a este nuevo miRNA (figura 5).

\section{DISCUSIÓN}

La transición del ovocito al embrión consiste en la inactivación del material genético proveniente del ovocito y la activación del genoma embrionario. Durante la activación del genoma embrionario los mRNAs maternos son degradados abruptamente, lo que permite una reorganización genética para promover la expresión de genes propios del embrión (Bettegowda y col 2008). Ahora bien, la competencia de un ovocito radica en soportar estos cambios abruptos de mRNAs que vienen desde la etapa de crecimiento (profase I), pasando por la metafase II y cul- 


\section{A \\ CROMOSOMA 8}

NW_003104074.1 Bos taurus_UMD_3.1

\begin{tabular}{|l|l|l|l|l|l|l|l|l|}
\hline & Exón 4 & & Exón 3 & Exón 2 & bta-mir-8548 & Exón 1 \\
\hline
\end{tabular}

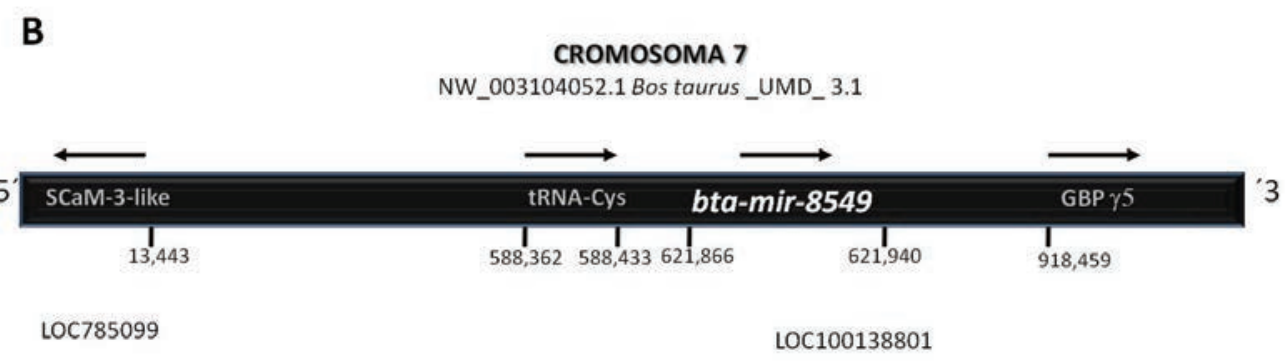

Figura 2. Localización cromosomal de los nuevos pre-miRNAs identificados. A. bta-mir-8548. B. bta-mir-8549.

Chromosomal location of the new pre-miRNAs identified. A. bta-mir-8548. B. bta-mir-8549.

Cuadro 2. Secuencia y localización dentro del genoma de Bos taurus de los nuevos pre-miRNAs identificados.

Sequence and location into Bos taurus genome of the newly identified pre-miRNAs.

\begin{tabular}{|c|c|c|c|c|c|c|}
\hline miRNA & Precursor miRNA 5' - '3 & $\begin{array}{c}\text { Tamaño } \\
\text { (nt) }\end{array}$ & $\begin{array}{l}\text { Localización } \\
\text { cromosoma ( } B . \\
\text { taurus) }\end{array}$ & $\begin{array}{c}\text { N'Acceso Gene } \\
\text { Bank }\end{array}$ & Inicio & Final \\
\hline bta-mir-8548 & GUCCCCUUUUCCUCCUGCCACCAACGUGAGGAUCAUGCUUUGAAAAGGAUUACCUUGGUGCAUUGC & 66 & 8 & NW_003104052.1 & 621866 & 621940 \\
\hline bta-mir-8549 & UUAUGGAGGUYAGUAGGUGGUAUUUAAAAGGGAGAUGAGUUCAUUUAUUCAUUUACAUGUUACCAUAUAUU & 84 & 7 & NW_003104074.1 & 587433 & 587359 \\
\hline
\end{tabular}

La secuencia del miRNA maduro aislado en la librería de miRNAs aparece subrayada.

A

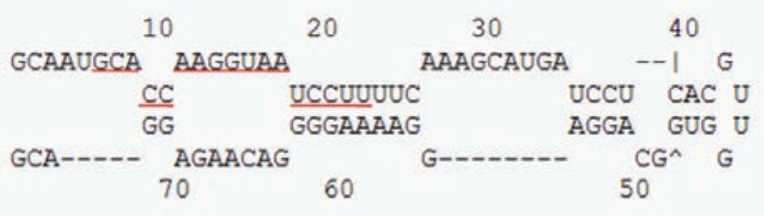

\section{B}

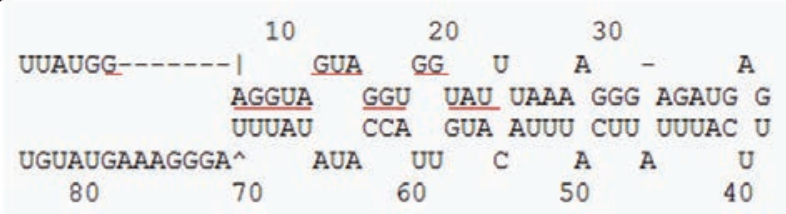

Figura 3. Estructura tallo-burbuja de los nuevos pre-miRNAs identificados. A. bta-mir-8548. B. bta-mir-8549. Para ambos casos en rojo se subraya la secuencia del miRNA maduro.

Stem-loop structure of the new pre-miRNAs identified. A. bta-mir-8548. B. bta-mir-8549. Red underline highlights the mature miRNA sequence. 

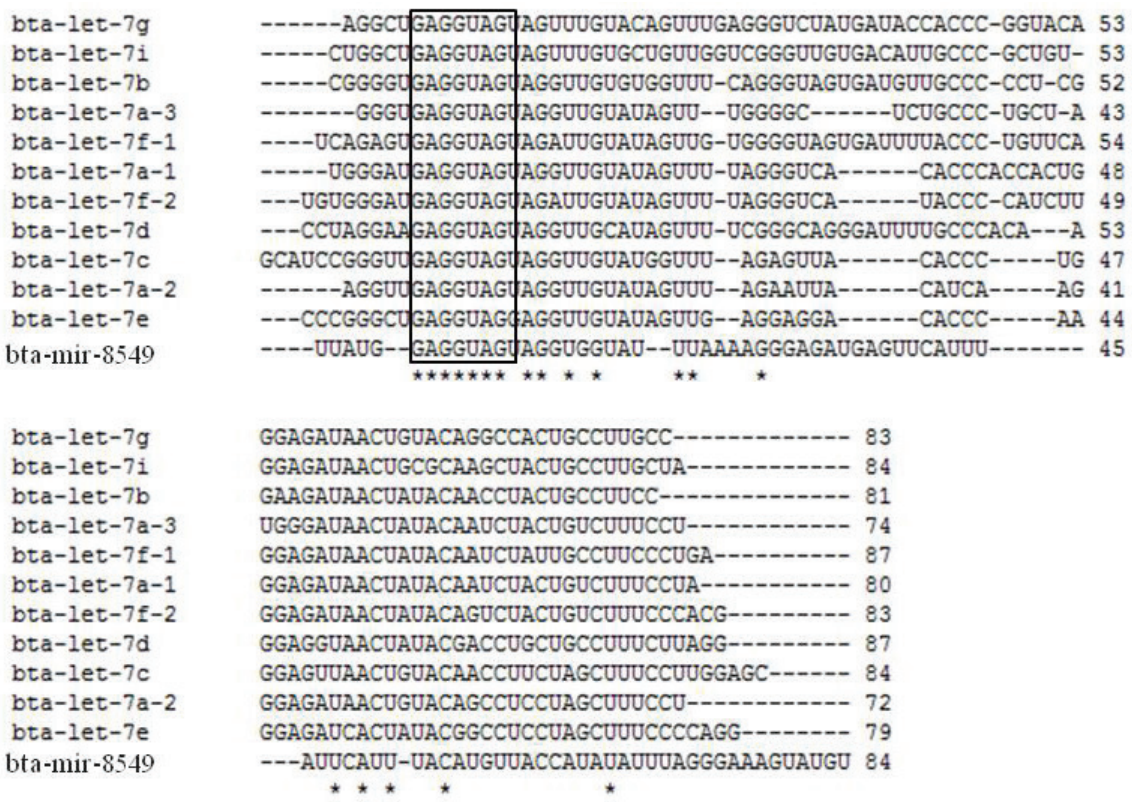

Figura 4. Alineación del pre-miRNA bta-mir-8549 contra los pre-miRNAs de la familia let-7 de Bos taurus tomando las secuencias ya reportadas en el miRBase para bta-let-7a (MIMAT003844), bta-let7-b (MIMAT0004331), bta-let7-c (MIMAT0004332), bta-let7-d (MIMAT0004332), bta-let7-e (MIMAT0004333), bta-let-7f(MIMAT0003519), bta-let-7g (MIMAT0003838), bta-let7-i (MIMAT0003851). El recuadro enmarca la secuencia conservada de los miRNAs de la familia let-7 en bovino. Los * señalan que en todos los casos se encuentra la misma base en la misma posición.

Alignment of the pre-miRNA bta-mir-8549 against sequences of pre-miRNAs into let-7 family of Bos taurus reported in miRBase to btalet-7a (MIMAT003844), bta-let7-b (MIMAT0004331), bta-let7-c (MIMAT0004332), bta-let7-d (MIMAT0004332), bta-let7-e (MIMAT0004333), bta-let-7f (MIMAT0003519), bta-let-7g (MIMAT0003838), bta-let7-i (MIMAT0003851). The box shows the conserved sequence of the miRNAs of the let-7 family in cattle. * indicates the same basis in the same position for every case.

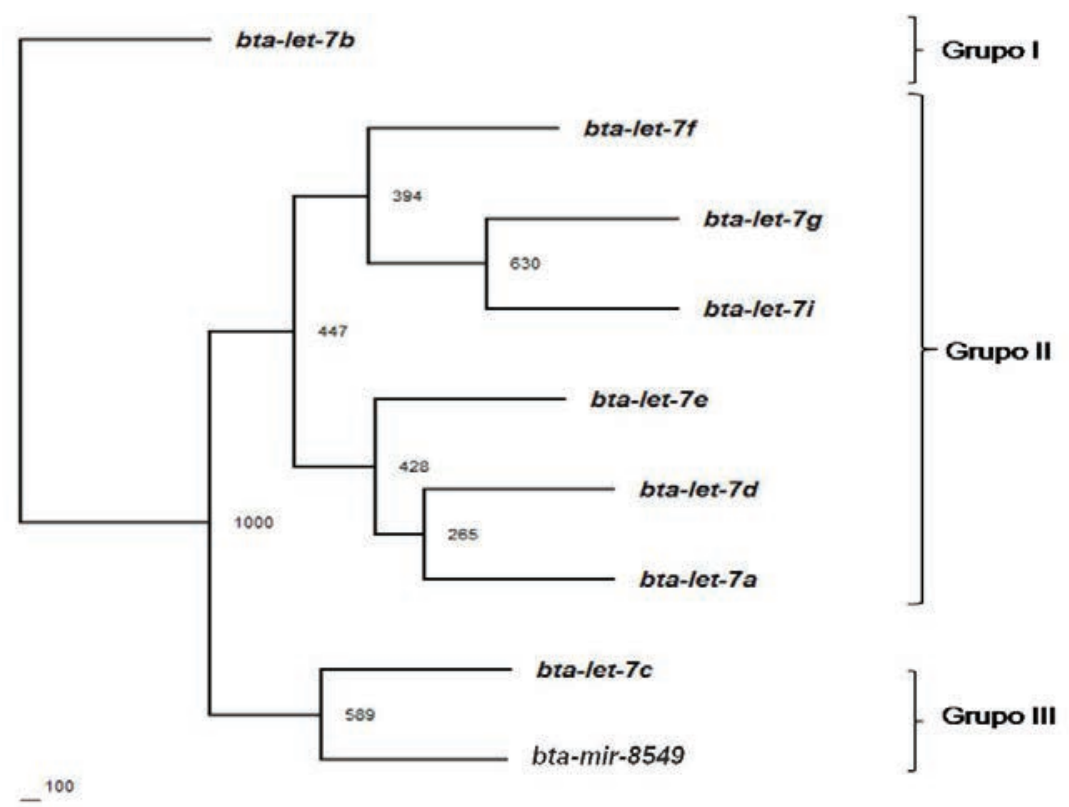

Figura 5. Filogenia de la familia de pre-miRNAs let-7 en bovino. El árbol consenso de mayoría fue construido conforme a las distancias genéticas generadas con el método de dos parámetros de Kimura usando el método Neighbour-joining. El largo de las ramas es proporcional al número de substituciones por sitio. Los números en los clados indican el remuestreo (bootstrap) derivado de 1000 réplicas.

Phylogeny of pre-miRNAs let-7 family in cattle. The majority consensus tree was designed according to the genetic distances generated by Kimura's two-parameter method with Neighbour-joining. Branch length is proportional to the number of substitutions per site. Numbers in clades indicate resampling (bootstrap) derived from 1000 replicates. 
minando en las etapas iniciales del desarrollo embrionario. Así que, el ovocito debe de poseer una regulación óptima a ese nivel, reprimiendo o expresando genes en cada una de estas etapas (De La Fuente 2006). Ya que el desarrollo de un ovocito competente es un proceso extremadamente complejo que involucra la regulación de genes a un nivel transcripcional y postranscripcional, los miRNA son excelentes candidatos para ser parte de la regulación de este proceso. Esto es claro en modelos como el ratón, pez cebra y trucha arcoíris (Giraldez y col 2006, Tang y col 2007, Ramachandra y col 2008), donde durante la activación del genoma embrionario se lleva a cabo de forma concomitante con la acción de los miRNAs al degradar mRNAs materno. Esto hace suponer que en el caso de mamíferos como las vacas el mecanismo sea parecido. Al respecto, Tesfaye y col (2009) hibridaron miRNAs provenientes de ovocitos inmaduros y maduros de vacas, con microarreglos de miRNAs de humano y ratón, y lograron aislar 59 microRNAs específicos del ganado bovino, los cuales evaluaron en ensayos de RT-PCR cuantitativa a lo largo de las diferentes etapas de la embriogénesis temprana. De estos, destacaron miR127 y miR-145, cuya expresión aumenta en la etapa de 8 células y decae en las etapas posteriores, justo durante la activación del genoma embrionario. Por su parte, Tripurani y col (2011) encontraron que en vacas el mRNA materno de ovario de recién nacido homeótico (NOBOX, por sus siglas en inglés) se degrada durante la activación del genoma embrionario debido a la unión del miR-196a en su extremo UTR'3 (Tripurani y col 2011). Algo similar observaron Lingenfelter y col (2011) para el mRNA materno bovino nucleoplasmina 2 (NPM2, por sus siglas en inglés) el cual es regulado por la unión en su UTR'3 del miR-181a (Lingenfelter y col 2011). Como se puede observar, aún es poco el conocimiento que se tiene sobre el mecanismo de acción de estos miRNAs en el proceso de la activación del genoma embrionario bovino. Teniendo en cuenta que la degradación de mRNAs maternos es un paso clave para que se dé la activación del genoma embrionario, es necesario conocer que tipos de miRNAs participan.

En este estudio, a partir de una genoteca de miRNAs de ovario bovino, se logró identificar dos nuevos miRNAs, el bta-mir-8548 y bta-mir-8549, cuyos miRNAs precursores mostraron una estructura tallo-burbuja estable, de acuerdo a los parámetros establecidos por el miRBase (Ambros y col 2003).

El bta-mir-8548, se ubicó en el cromosoma 8 en una región intrónica entre los exones 1 y 2 del gen MRSA, el cual codifica para la proteína metionina sulfóxido reductasa A (MRSA, por sus siglas en inglés), que actúa como una enzima antioxidante al reparar los residuos de metioninas oxidadas durante el estrés oxidativo (Hansel y col 2005). La expresión de este gen se da en casi todas las células y tejidos de la mayoría de los mamíferos superiores (Weissbach y col 2005), por lo que no es de sorprender que se encontrara en una genoteca proveniente de ovario, como la de este estudio. Lo que sorprende es que exista un
miRNA codificado en uno de los intrones de este gen. Se sabe que durante el crecimiento y maduración del ovocito, este gameto está expuesto a la acción de especies reactivas del oxígeno (ROS, por sus siglas en inglés). Los ROS, en condiciones apropiadas, son necesarios para que el desarrollo de la foliculogénesis se dé en forma adecuada (Hancock y col 2001). Un aumento en la concentración de estos radicales libres está asociado con un arresto de la meiosis del ovocito en crecimiento (Nakamura y col 2002), pérdida de la zona pelúcida y disminución de gránulos corticales (Goud y col 2008). Así que la neutralización de los ROS es indispensable para que este proceso celular se dé correctamente. Esto hace suponer que el bta-mir-8548 puede estar implicado, al igual que MRSA, en el control del estrés oxidativo durante la maduración del ovocito.

El bta-mir-8549, se ubicó en el cromosomal 7 en la región intergénica entre los genes tRNA-Cys y GBPg5. La secuencia consenso del bta-mir-8549 maduro, mostró estar relacionada con los miRNA de la familia let-7, la cual es una familia de miRNAs heterocrónicos muy conservada en organismos como Drosophila y en el ser humano (Pasquinelli y col 2000). Tanto en C. elegans como en ratón, este tipo de miRNAs se unen de forma complementaria al 3’UTR del gen lin-41 con el único fin de inhibir su expresión al impedir su traducción (Slack y col 2000, Schulman y col 2005). lin-41, es también es un gen heterocrónico, en ratón su expresión se observa de forma temporal durante el desarrollo embrionario (Schulman y col 2005). Aunque en el ganado bovino el gen lin-41 aún no se identifica, ya están reportados los miRNAs bta-let7a, bta-let-7b, bta-let-7c, bta-let-7d, bta-led-7c, bta-led$7 f$, bta-let-7g y bta-let-7i (Coutinho y col 2007, Tesfaye y col 2009). Estudios realizados por Miles y col (2012) muestran que los miRNAs bta-let-7b, bta-let-7i, bta-let$7 f$ y bta-let-7e, son altamente expresados en el complejo cumulus-ovocito (COC, por sus siglas en inglés), y relacionan su función sobre la expresión de los genes $M Y C$ y WEE1A involucrados en los procesos de mitosis y meiosis del ovocito durante su maduración (Miles y col 2012). Esto sugiere que es probable que el bta-mir-8549 esté presente en el ovario con la finalidad de regular la expresión de genes que participan con la maduración del ovocito.

En conclusión, bta-mir-8548 y bta-mir-8549 son nuevos miRNAs presentes en el ovario bovino. Dado que la genoteca de miRNAs se obtuvo de la gónada completa, aún es muy aventurado suponer que estos miRNAs pudieran participar en el activación del genoma embrionario. Sin embargo, es claro que están presentes en el ovario y pudieran participar en alguno de los procedimientos celulares que en esta gónada se llevan a cabo.

\section{AGRADECIMIENTOS}

Al PROMEP-SEP-México por el apoyo otorgado OF-09415. L. A. Muñoz-Bañales fue becaria del CONACyT-México (Reg. 36012 ). 


\section{REFERENCIAS}

Ambros V, B Bartel, DP Bartel, CB Burge, JC Carrington, X Chen, G Dreyfuss, SR Eddy, S Griffiths-Jones, M Marshall, M Matzke, G Ruvkun, T Tuschl. 2003. A uniform system for microRNA annotation. RNA 9, 277-279.

Artzi S, A Kiezun, N Shomron. 2008. miRNAminer: a tool for homologous microRNA gene search. BMC Bioinformatics 9, 39 .

Bettegowda A, KB Lee, GW Smith. 2008. Cytoplasmic and nuclear determinants of the maternal-to-embryonic transition. Reprod Fert Develop 20, 45-53.

Bushati N, SM Cohen. 2007. microRNA functions. Annu Rev Cell Dev Biol 23, 175-205.

Coutinho LL, LK Matukumalli, TS Sonstegard, CP Van Tassell, LC Gasbarre, AV Capuco, TP Smith. 2007. Discovery and profiling of bovine microRNAs from immune-related and embryonic tissues. Physiol Genomics 29, 35-43.

De La Fuente R. 2006. Chromatin modifications in the germinal vesicle (GV) of mammalian oocytes. Dev Biol 292, 1-12.

Filipowicz W, SN Bhattacharyya, N Sonenberg. 2008. Mechanisms of post-transcriptional regulation by microRNAs: are the answers in sight? Nat Rev Genet 9, 102-114.

Giraldez AJ, Y Mishima, J Rihel, RJ Grocock, S Van Dongen, K Inoue, AJ Enright AF Schier. 2006. Zebrafish MiR-430 promotes deadenylation and clearance of maternal $\mathrm{mR}$ NAs. Science 312, 75-79.

Goud AP, PT Goud, MP Diamond, B Gonik HM Abu-Soud. 2008. Reactive oxygen species and oocyte aging: role of superoxide, hydrogen peroxide, and hypochlorous acid. Free Radic Biol Med 44, 1295-1304.

Hancock JT, R Desikan, SJ Neill. 2001. Role of reactive oxygen species in cell signalling pathways. Biochem Soc Trans 29, 345-350.

Hansel A, SH Heinemann, T Hoshi. 2005. Heterogeneity and function of mammalian MSRs: enzymes for repair, protection and regulation. Biochim Biophys Acta 1703, 239-247.

Kimura M. 1980. A simple method for estimating evolutionary rates of base substitutions through comparative studies of nucleotide sequences. J Mol Evol 16, 111-120.

Kozomara A, S Griffiths-Jones. 2011. miRBase: integrating microRNA annotation and deep-sequencing data. Nucleic Acids Res 39, 152-157.

Li L, P Zheng J Dean. 2010. Maternal control of early mouse development. Development 137, 859-870.

Lingenfelter BM, SK Tripurani, J Tejomurtula, GW Smith, J Yao. 2011. Molecular cloning and expression of bovine nucleoplasmin 2 (NPM2): a maternal effect gene regulated by miR-181a. Reprod Biol Endocrinol 9, 40.

Miles JR, TG McDaneld, RT Wiedmann, RA Cushman, SE Echternkamp, JL Vallet, TPL Smith. 2012. MicroRNA expression profile in bovine cumulus-oocyte complexes: Possible role of let-7 and miR-106a in the development of bovine oocytes. Anim Reprod Sci 130, 16-26.

Nakamura Y, Y Yamagata, N Sugino, H Takayama, H Kato.
2002. Nitric oxide inhibits oocyte meiotic maturation. Biol Reprod 67, 1588-1592.

Pasquinelli AE, BJ Reinhart, F Slack, MQ Martindale, MI Kuroda, B Maller, DC Hayward, EE Ball, B Degnan, P Muller, J Spring, A Srinivasan, M Fishman, J Finnerty, J Corbo, M Levine, P Leahy, E Davidson, G Ruvkun. 2000. Conservation of the sequence and temporal expression of let-7 heterochronic regulatory RNA. Nature 408, 86-89.

Ramachandra RK, M Salem, S Gahr, CE Rexroad, J Yao. 2008. Cloning and characterization of microRNAs from rainbow trout (Oncorhynchus mykiss): Their expression during early embryonic development. Bmc Developmental Biology 8, 41.

Retief JD. 2000. Phylogenetic analysis using PHYLIP. Methods Mol Biol 132, 243-258.

Saitou N, M Nei. 1987. The neighbor-joining method: a new method for reconstructing phylogenetic trees. Mol Biol Evol 4, 406-425.

Schulman BR, A Esquela-Kerscher FJ Slack. 2005. Reciprocal expression of lin-41 and the microRNAs let-7 and mir-125 during mouse embryogenesis. Dev Dyn 234, 1046-1054.

Schultz RM. 1993. Regulation of zygotic gene activation in the mouse. Bioessays 15, 531-538.

Slack FJ, M Basson, Z Liu, V Ambros, HR Horvitz G Ruvkun. 2000. The lin-41 RBCC gene acts in the C. elegans heterochronic pathway between the let-7 regulatory RNA and the LIN-29 transcription factor. Mol Cell 5, 659-669.

Tang F, M Kaneda, D O'Carroll, P Hajkova, SC Barton, YA Sun, C Lee, A Tarakhovsky, K Lao MA Surani. 2007. Maternal microRNAs are essential for mouse zygotic development. Genes Dev 21, 644-648.

Telford NA, AJ Watson, GA Schultz. 1990. Transition from maternal to embryonic control in early mammalian development: a comparison of several species. Mol Reprod Dev 26, 90-100.

Tesfaye D, D Worku, F Rings, C Phatsara, E Tholen, K Schellander, M Hoelker. 2009. Identification and expression profiling of microRNAs during bovine oocyte maturation using heterologous approach. Mol Reprod Dev 76, 665677.

Thompson JD, DG Higgins, TJ Gibson. 1994. CLUSTAL W: improving the sensitivity of progressive multiple sequence alignment through sequence weighting, position-specific gap penalties and weight matrix choice. Nucleic Acids Res 22, 4673-4680.

Tripurani SK, KB Lee, G Wee, GW Smith, J Yao. 2011. MicroRNA-196a regulates bovine newborn ovary homeobox gene (NOBOX) expression during early embryogenesis. BMC Dev Biol 11, 25.

Weissbach H, L Resnick, N Brot. 2005. Methionine sulfoxide reductases: history and cellular role in protecting against oxidative damage. Biochim Biophys Acta 1703, 203-212.

Zuker M. 2003. Mfold web server for nucleic acid folding and hybridization prediction. Nucleic Acids Res 31, 34063415. 